PROCEEDINGS OF THE

AMERICAN MATHEMATICAL SOCIETY

Volume 131, Number 1, Pages 77-82

S 0002-9939(02)06652-2

Article electronically published on May 22, 2002

\title{
A NOTE ON A LEMMA OF SHIMURA
}

\author{
ZE-LI DOU
}

(Communicated by Dennis A. Hejhal)

\begin{abstract}
The subject of this paper is a quaternion algebra over a quadratic extension of a totally real algebraic number field. We generalize an important technical lemma of Shimura, on which a certain period of automorphic forms depends.
\end{abstract}

\section{INTRODUCTION}

We explain the background of this note by considering the simplest case, $F=\mathbb{Q}$. Let $B$ be an indefinite quaternion algebra over $\mathbb{Q}$, and let $f$ be a $\overline{\mathbb{Q}}$-rational eigenform of an even positive weight with respect to $B$. In an important article Sh82, Shimura attaches to $f$ two fundamental periods, $u_{+}(f)$ and $u_{-}(f)$, by cohomological means. On the other hand, he also considers a theta lift $I(z, f)$ of $f$, which is a half-integral weight elliptic modular form. Shimura then shows that the coefficients of $I(z, f)$ can be expressed in terms of a certain period of $f$, which is defined via an integral. This integral turns out to be essentially $u_{+}(f)$ when $f$ is primitive (and hence truly a period). The theta correspondence and the integral version of the period should not be seen as mere redundancies, however. Important arithmetic consequences (for instance the algebraicity of various period relations) follow, in fact, from this wider set-up.

A key technical lemma, Lemma 2.1 of Sh82, establishes the convergence of the integral defining the period and describes its behavior with respect to the variables. In view of what we have just said, this lemma occupies a pivotal position in the development of Shimura's theory.

The foregoing theory has been vastly extended, largely due to the work of Shimura himself. Fundamental periods have been considered in the setting of a totally real number field $F$, and sweeping and precise conjectures on their behavior have been formulated. See [Sh88] and [Sh90]; see also [D3]. The theta correspondence has also been extended by Shimura; see for example [Sh88] again. (Indeed, already in Sh82, the setting of the theta lift is somewhat more general than the form spoken of earlier. For more background and related works, one can also consult [Sh80], [Sh81I], [Sh81II], and [He85].)

Received by the editors February 14, 2000 and, in revised form, August 22, 2001

2000 Mathematics Subject Classification. Primary 11F30, 11F41, 11F55, 11F67.

Key words and phrases. Quaternionic automorphic forms, periods of automorphic forms, theta correspondence.

Research for this article was partially supported by NSA Grant MDA904-97-1-0109 and by the Texas Christian University Research Fund.

(C)2002 American Mathematical Society 
In view of recent progress on Shimura's period conjectures, it has now become necessary to consider similar lifts with respect to a totally quadratic extension $E / F$. This is the setting of the author's papers [D1] and [D2]. A generalization of Shimura's Lemma was called for there. In an effort to keep his articles as short as possible, however, the author opted to relegate the generalized lemma's proof to a later publication.

Our aim in the present note is to give a precise statement of this generalized lemma together with a detailed proof.

Though technically more complex, the outline of our proof will basically follow that of Shimura. The author is optimistic that this will also be the case in certain other settings, and hopes to present some such applications (growing out of this paper) in the near future.

We formulate our generalization of Shimura's Lemma in Section 1; its proof is then explained in Section 2.

\section{Generalization of Shimura's Lemma}

In this section we present an analogue of Shimura's Lemma. Let $F$ be a totally real algebraic number field, and let $B$ be a quaternion algebra over $F$. Let $E / F$ be a totally real quadratic extension, and $\tau$ a generator of $\operatorname{Gal}(E / F)$. Define

$$
B_{E}=B \otimes_{F} E \text {. }
$$

Then $B_{E}$ is a quaternion algebra over $E$. We extend $\tau$ to $B_{E}$ by putting

$$
(b \otimes e)^{\tau} \stackrel{\text { def }}{=} b \otimes e^{\tau}
$$

for all $e \in E$. We shall consider matrix-valued automorphic forms with respect to $B_{E}$, and define periods of such forms.

Let $\mathrm{N}$ and $\mathrm{Tr}$ be the norm and trace for $B_{E}$. Define

$$
V=\left\{x \in B_{E} \mid x^{\tau}=-x^{*}\right\}
$$

Then $V$ is a vector space over $F$ of dimension 4 . It can be checked easily that $\mathrm{N}$ is an $F$-valued quadratic form on the vector space $V$. Further, given $a \in B_{E}$, we have

$$
\mathrm{N}\left(a^{\tau} x a^{*}\right)=\mathrm{N}^{\prime}(a) \mathrm{N}(x), \quad \text { where } \quad \mathrm{N}^{\prime}(a) \stackrel{\text { def }}{=} \mathrm{N}_{E / F}(\mathrm{~N}(a)) .
$$

To consider automorphic forms, it is necessary for us to keep in view both $F$ and $E$. Thus we fix notation as follows. Let $\mathbf{a}$ and $\mathbf{h}$ denote the sets of archimedean and finite primes in $F$, respectively. Following Shimura, the set of archimedean primes in $E$ is written $J(E)$. We identify certain subsets of a and pair them with corresponding subsets of $J(E)$, which consist of their extensions. Let $\delta$ denote the set of primes $v \in \mathbf{a}$ which are unramified in $B$, and $\delta^{\prime}=\mathbf{a}-\delta$ the set of those ramified in $B$. The subsets of $J(E)$ consisting of extensions of primes in $\delta$ and $\delta^{\prime}$ are then denoted by $\zeta$ and $\zeta^{\prime}$, respectively. Using these notations, we have $\mathbb{R}$-linear isomorphisms

$$
B_{\mathbf{a}} \cong \mathrm{M}_{2}(\mathbb{R})^{\delta} \times \mathbb{H}^{\delta^{\prime}} \text { and }\left(B_{E}\right)_{\mathbf{a}} \cong \mathrm{M}_{2}(\mathbb{R})^{\zeta} \times \mathbb{H}^{\zeta^{\prime}}
$$

Here the subscript a indicates the infinite part of the adelized space under consideration. For each $v \in \mathbf{a}$, we fix, once and for all, an extension $u \in J(E)$. The collection of these chosen $u$ is written $\iota$. Further, we denote by $\eta$ and $\eta^{\prime}$, respectively, the subsets of $\iota$ corresponding to $\delta$ and $\delta^{\prime}$. We assume $\zeta \neq \emptyset$ throughout. 
If $k \in \mathbb{Z}^{\iota}$ is a weight such that $k_{u} \geq 1$ for $u \in \eta$ and $k_{u} \geq 2$ for $u \in \eta^{\prime}$, then we define as usual a representation on $\left(B_{E}^{\times}\right)_{\mathbb{A}}$ by

$$
\sigma(\alpha)=\bigotimes_{u \in \eta^{\prime}} \sigma_{k_{u}-2}\left(\alpha_{u}\right), \quad \forall \alpha \in\left(B_{E}^{\times}\right)_{\mathbb{A}}
$$

A more detailed description of the representation is given on p. 5 of [D1]. The representation space for $\sigma$ will be denoted by $\mathcal{X}$. It may be identified with $\bigotimes_{u \in \eta^{\prime}} \mathbb{C}^{k_{u}-1}$.

We introduce some more notation. Let

$$
\begin{aligned}
\mathcal{G}^{\prime}=\left(B_{E}\right)^{\times}, & \mathcal{G}_{\mathbf{a}+}^{\prime}=\left\{x \in \mathcal{G}_{\mathbf{a}}^{\prime} \mid N(x) \gg 0\right\}, \\
\mathcal{G}_{\mathbb{A}+}^{\prime}=\left\{x \in \mathcal{G}_{\mathbb{A}}^{\prime} \mid x_{\mathbf{a}} \in \mathcal{G}_{\mathbf{a}+}^{\prime}\right\}, & \mathcal{G}_{1}^{\prime}=\left\{x \in \mathcal{G}^{\prime} \mid \mathrm{N}(x)=1\right\} .
\end{aligned}
$$

Thus the representation in (1) is $\sigma: \mathcal{G}_{\mathbb{A}}^{\prime} \rightarrow G L(\mathcal{X})$.

We now define $\operatorname{End}(\mathcal{X})$-valued holomorphic automorphic forms weight $k+\tau k$ on $H^{\zeta}$, where $k \in \mathbb{Z}^{\iota}$ as above. Given a mapping $f: H^{\zeta} \rightarrow \operatorname{End}(\mathcal{X})$, we define another mapping of the same kind by the following formula:

$$
\left(f \|_{k+\tau k} \alpha\right)(w)=j(\alpha, w)^{-k \eta-\tau(k \eta)} \sigma\left(\mathrm{N}(\alpha)^{\frac{1}{2}} \alpha^{-1}\right) f(\alpha w) \sigma\left(\mathrm{N}(\alpha)^{-\frac{\tau}{2}} \alpha^{\tau}\right) .
$$

Let $\Gamma$ be a congruence subgroup of $\mathcal{G}_{1}^{\prime}$. Then the space of holomorphic automorphic forms of weight $k+\tau k$ with respect to $\Gamma$ is the set of all holomorphic mappings $f: H^{\zeta} \rightarrow \operatorname{End}(\mathcal{X})$ such that $f \| \alpha=f$ for all $\alpha \in \Gamma$, and in the case of $B_{E}=\mathrm{M}_{2}(E)$, that they also satisfy the usual cusp condition. This space is denoted by $\mathcal{S}_{k+\tau k}(\Gamma)$. The union of such spaces over all congruence subgroups is denoted by $\mathcal{S}_{k+\tau k}\left(B_{E}\right)$.

Next we generalize the Petersson inner product to our setting. Denote by $d_{H}^{\zeta} w$ the Haar measure on $H^{\zeta}$. More explicitly, put

$$
d_{H}^{\zeta} w=(2 i)^{-\#(\zeta)} \prod_{u \in \zeta} \operatorname{Im}\left(w_{u}\right)^{-2} d \bar{z}_{u} \wedge d z_{u}
$$

If $f$ and $g$ are two cusp forms with respect to $\Gamma$, we then define

$$
\langle f, g\rangle=\operatorname{vol}(D)^{-1} \int_{D} \operatorname{Tr}\left(\overline{{ }^{t} f(w)} g(w)\right) \operatorname{Im}(w)^{k \eta+\tau(k \eta)} d_{H}^{\zeta} w .
$$

Here $D \stackrel{\text { def }}{=} \Gamma \backslash H^{\zeta}$ and $\operatorname{vol}(D) \stackrel{\text { def }}{=} \int_{D} d_{H}^{\zeta} w$. This definition is independent of the choice of $\Gamma$.

We prepare for the statement of Theorem 1 by recognizing certain identifications regarding the localizations of $B_{E}$ and $B$. Let $v \in \delta$ and let $u$ be the extension of $v$ in $\iota$. Then we have

$$
\left(B_{E}\right)_{v}=\left(B_{E}\right)_{u} \times\left(B_{E}\right)_{\tau u}=B_{v} \times B_{v}=\mathrm{M}_{2}(\mathbb{R}) \times \mathrm{M}_{2}(\mathbb{R}) .
$$

Consequently, for $x, y \in B_{v}$ we have $(x, y)^{\tau}=(y, x)$. Thus we further identify

$$
V_{v}=\left\{\left(x,-x^{*}\right) \mid x \in B_{v}\right\}=B_{v} .
$$

When $v \in \delta^{\prime}$, a similar consideration shows that $\left(B_{E}\right)_{v}=\mathbb{H} \times \mathbb{H}$.

Let an element $0 \neq \alpha \in V$ and a congruence subgroup $\Delta \subset \mathcal{G}_{1}^{\prime}$ be given. Put

$$
K_{\alpha}=\left\{\xi \in B_{E} \mid \xi \alpha=\alpha \xi^{\tau}\right\}, \Delta_{\alpha}=\Delta \cap K_{\alpha}, \text { and } D_{\alpha}=\Delta_{\alpha} \backslash H^{\zeta} .
$$

Then it can easily be checked that $K_{\alpha}$ is a quaternion algebra over $F$, and we have

$$
\Delta_{\alpha}=\left\{\gamma \in \Delta \mid \gamma^{*} \alpha \gamma^{\tau}=\gamma^{-1} \alpha \gamma^{\tau}=\alpha\right\}
$$

Write $\mathcal{S}=\mathrm{SL}_{2}(\mathbb{R})^{\zeta}$. Then $\Delta_{\alpha}$ can be viewed as a subgroup of $\mathcal{S}$. Put $J_{\alpha}=\{g \in$ $\left.\mathcal{S} \mid g \alpha=\alpha g^{\tau}\right\}$. 
Given a cusp form $h \in \mathcal{S}_{k+\tau k}\left(B_{E}\right)$, a period of $h$ is then defined as follows:

$$
P(h, \alpha, \Delta)=\int_{\Delta_{\alpha} \backslash J_{\alpha}}[\alpha, p w]^{k \eta} \operatorname{Tr}\left({ }^{t} \overline{\sigma(\alpha)} h(p w)\right) d\left(\Delta_{\alpha} p\right),
$$

where $w \in H^{\zeta}$.

Theorem 1. The integral

$$
\int_{\Delta_{\alpha} \backslash J_{\alpha}}[\alpha, p w]^{k \eta} \operatorname{Tr}\left({ }^{t} \overline{\sigma(\alpha)} h(p w)\right) d\left(\Delta_{\alpha} p\right)
$$

is convergent, and, in fact, is a constant independent of $w$. Moreover, the integral vanishes unless $\mathrm{N}(\alpha)_{v}<0$ for every $v \in \delta$.

This is the exact analogue of Shimura's Lemma (see Lemma 2.1 of [Sh82]) in our setting.

\section{Demonstration}

In this section we prove Theorem 1. We follow the same outline of Shimura's proof. However, the present situation is technically more complicated.

Recall from the last section that we have $\left(B_{E}\right)_{\mathbf{a}} \cong B_{\mathbf{a}} \times B_{\mathbf{a}}$, hence $V_{\mathbf{a}}=$ $\left\{\left(x,-x^{*}\right) \mid x \in B_{\mathbf{a}}\right\}$. Thus, writing $\alpha=\left(b,-b^{*}\right)$ with some $b \in B_{\mathbf{a}}$, we see that

$$
K_{\alpha} \otimes_{\mathbb{Q}} \mathbb{R}=\left\{\left(x, b^{-1} x b\right) \mid x \in B_{\mathbf{a}}\right\} \quad \text { and } \quad J_{\alpha}=\left\{\left(x, b^{-1} x b\right) \mid x \in \mathrm{SL}_{2}(\mathbb{R})^{\delta}\right\} .
$$

Therefore $\Delta_{\alpha} \backslash J_{\alpha}$ is compact. This gives the convergence.

For notational convenience, let us write the integral $(2)$ as $\phi(w)$. Then $\phi(w)$ is holomorphic in $w$, and for every $p \in J_{\alpha}$ we have $\phi(p w)=\phi(w)$. Let $s \in H^{\zeta}$ be any point and set $T=\left\{g \in \mathrm{SL}_{2}(\mathbb{R})^{\delta} \mid g b s=b s\right\}$. Clearly $T \cong \mathrm{SO}(2)^{\delta}$. Then

$$
\phi((g z, s))=\phi\left(\left(g, b^{-1} g b\right)(z, s)\right)=\phi(z, s)
$$

for all $g \in \mathrm{SL}_{2}(\mathbb{R})^{\delta}$. Therefore $\phi((z, s))$ is independent of $z$, and hence is a function of $s$ only. Let us write it as $\psi(s)$. But

$$
\psi(g s)=\phi\left(\left(b g b^{-1}, g\right)(z, s)\right)=\phi(z, s)=\psi(s) .
$$

Thus we have shown that $\phi(w)$ is indeed a constant.

To consider the vanishing, suppose $\mathrm{N}(\alpha)_{v}>0$ for some $v \in \delta$. There exists some $w_{u}$ such that $w_{u}=\alpha_{u}\left(w_{\tau u}\right)$ holds. For this $w_{u}$ we have

$$
[\alpha, w]_{u}=\left(\begin{array}{ll}
-1 & w_{u}
\end{array}\right) \alpha_{u}\left(\begin{array}{c}
w_{\tau u} \\
1
\end{array}\right)=0 .
$$

Since $\phi(w)=[\alpha, w]^{k \eta} \int_{\Delta_{\alpha} \backslash J_{\alpha}} j(p, w)^{-k \eta-\tau(k \eta)} h_{\alpha}(p w) d\left(\Delta_{\alpha} p\right)$, this integral is 0 as well.

We now consider the case $\mathrm{N}(\alpha)=0$. This happens only when $B_{E}=\mathrm{M}_{2}(E)$. In this case we may assume $B=\mathrm{M}_{2}(F)$. For $\xi \in \mathrm{M}_{2}(E)$, let us write ${ }^{\tau} \xi$ for the entrywise action of $\tau$. Then we have $\xi^{\tau}=\omega \cdot{ }^{\tau} \xi \omega^{-1}$ with $\omega \in \mathrm{GL}_{2}(E)$. A straightforward calculation shows that ${ }^{\tau} \omega \omega \in F$. Call this element $c$. Now $B$ can be characterized as $B=\left\{x \in \mathrm{M}_{2}(E) \mid x^{\tau}=x\right\}=\left\{x \in \mathrm{M}_{2}(E) \mid x \omega=\omega \cdot{ }^{\tau} x\right\}$. Since $\alpha^{\tau}=-\alpha^{*}$, we have

$$
K_{\alpha}=\left\{\xi \in B_{E} \mid \xi \cdot \alpha \omega=\alpha \omega \cdot{ }^{\tau} \xi\right\}
$$


Put $\eta=\alpha \omega$. Then $\eta^{*}=\omega^{*} \alpha^{*}=\operatorname{det}(\omega) \omega^{-1} \cdot\left(-\alpha^{\tau}\right)=-c^{-1} \operatorname{det}(\omega)^{\tau}(\alpha \omega)=\mu \cdot{ }^{\tau} \eta$. Therefore $\operatorname{det}(\eta)=0$. The set of such $\eta$ is stable under $\eta \mapsto^{\tau} \gamma \cdot \eta \gamma^{*}$, and is given by

$$
A=\left\{\left(\begin{array}{cc}
p & q \\
r & \mu p^{\tau}
\end{array}\right): \mu p p^{\tau}=q r, q=-\mu q^{\tau}, r=-\mu r^{\tau}\right\} .
$$

If $q=0$, then $p=0$ as well, and hence $\eta=\left(\begin{array}{ll}0 & 0 \\ r & 0\end{array}\right)$. Suppose $q \neq 0$. Then we note that

$$
\left(\begin{array}{cc}
1 & 0 \\
-q^{-1} s & 1
\end{array}\right)\left(\begin{array}{ll}
p & q \\
r & s
\end{array}\right)\left(\begin{array}{ll}
1 & 0 \\
* & 1
\end{array}\right)=\left(\begin{array}{cc}
* & q \\
* & 0
\end{array}\right)
$$

Since this is in $A$, the only possibility is $\left(\begin{array}{ll}0 & q \\ 0 & 0\end{array}\right)$. Therefore we conclude that there exists a $\gamma \in B_{E}$, such that $\mathrm{N}(\gamma)=1$ and $\gamma^{-1} \eta \cdot{ }^{\tau} \gamma=\left(\begin{array}{cc}0 & q \\ 0 & 0\end{array}\right)$.

Now if $\xi \in K_{\alpha}$, then $\gamma^{-1} \xi \gamma \cdot \gamma^{-1} \eta \cdot{ }^{\tau} \gamma=\gamma^{-1} \eta \cdot{ }^{\tau} \xi \cdot{ }^{\tau} \gamma=\gamma^{-1} \eta \cdot{ }^{\tau} \gamma \cdot{ }^{\tau}\left(\gamma^{-1} \xi \gamma\right)$. Therefore we have the following:

$$
\begin{aligned}
\gamma^{-1} K_{\alpha} \gamma & =\left\{x \in B_{E}: x \cdot\left(\begin{array}{ll}
0 & q \\
0 & 0
\end{array}\right)=\left(\begin{array}{ll}
0 & q \\
0 & 0
\end{array}\right) \cdot{ }^{\tau} x\right\} \\
& =\left\{\left(\begin{array}{cc}
a & b \\
0 & a^{\tau}
\end{array}\right): a \in E, b \in E\right\}
\end{aligned}
$$

Thus

$$
\gamma^{-1} \Delta_{\alpha} \gamma \subset\left\{\left(\begin{array}{cc}
a & b \\
0 & a^{\tau}
\end{array}\right): a a^{\tau}=1\right\}
$$

Finally

$$
\gamma^{-1} J_{\alpha} \gamma=\left\{\left(\left(\begin{array}{cc}
a & b \\
0 & a^{-1}
\end{array}\right),\left(\begin{array}{cc}
a & b^{\prime} \\
0 & a^{-1}
\end{array}\right)\right)\right\} \subset \mathrm{SL}_{2}(\mathbb{R})^{\mathbf{a}} \times \mathrm{SL}_{2}(\mathbb{R})^{\mathbf{a}} .
$$

In this fashion, the integral over $\Delta_{\alpha} \backslash J_{\alpha}$ may be transformed into an iterated integral $\int_{U \backslash\left(\mathbb{R}^{\times}\right)^{\mathrm{a}}} \int_{\mathbb{R}^{J(E)} / L} h$, where $U$ is a set of units, and $L$ is a lattice. Since $h$ is a cusp form, the integral is 0 . This completes the proof of Theorem 1.

\section{ACKNOWLEDGMENT}

The author thanks Professor Goro Shimura for suggesting the problem, and for his generous advice and help. The author is grateful to Professor Dennis Hejhal for his support at a crucial juncture. Finally, he thanks the referee for some corrections, and, especially, for his suggestions on stylistic improvements.

\section{REFERENCES}

[D1] Z.-L. Dou, On a theta correspondence with respect to a quadratic extension, J. Number Theory 79 (1999), 1-18. MR 2000j:11063

[D2] - Theta correspondence and Hecke operators relative to a quadratic extension, Acta Arithmetica XCIII.3 (2000), 237-255. MR 2001f:11074

[D3] On the fundamental periods of Hilbert modular forms, Transactions of the American Mathematical Society 346 (1994), 147-158. MR 95b:11052

[He85] D. A. Hejhal, A classical approach to a well-known correspondence on quaternion groups, Lecture Notes in Math. 1135 (1985), 127-196, Springer-Verlag. MR 87h:11045

[Sh80] G. Shimura, The arithmetic of certain zeta functions and automorphic forms on orthogonal groups, Ann. Math. 111 (1980), 313-375. MR 81g:10041

[Sh81I] On certain zeta functions attached to two Hilbert modular forms, I, Ann. Math. 114 (1981), 127-164. MR 83d:10036a 
ZE-LI DOU

[Sh81II] _ On certain zeta functions attached to two Hilbert modular forms, II, Ann. Math. 114 (1981), 569-607. MR 83d:10036b

[Sh82] - The periods of certain automorphic forms of arithmetic type, J. Fac. Sci. Univ. Tokyo (Sect. IA) 28 (1982), 605-632. MR 84f:10040

[Sh88] - On the critical values of certain Dirichlet series and the periods of automorphic forms, Invent. Math. 94 (1988), 245-305. MR 90e:11069

[Sh90] On the fundamental periods of automorphic forms of arithmetic type, Invent. Math. 102 (1990), 399-428. MR 91k:11041

Department of Mathematics, Texas Christian University, P. O. Box 298900, Fort Worth, TEXAs 76129

E-mail address: z.dou@tcu.edu 